\title{
SEGURIDAD ALIMENTARIA Y PRODUCCIÓN PECUARIA CAMPESINA: EL CASO DE LA LOCALIDAD RURAL DE SUMAPAZ
}

\author{
Omar Felipe Giraldo* \\ * Médico Veterinario. Universidad Nacional de Colombia. Magister (c) en Desarrollo \\ Rural. Universidad Nacional de Costa Rica. Correo Electrónico: \\ omarfgiraldo@hotmail.com
}

Manizales, 2008-11-17 (Rev. 2008-12-06)

\section{RESUMEN}

El artículo aborda el tema de la seguridad alimentaría y la producción pecuaria campesina a partir del estudio del caso de la Localidad del Sumapaz, la cual pertenece al área rural de Bogotá D.C. Este territorio afronta una grave situación de inseguridad alimentaría de acuerdo con información de estudios previos. Mediante la aproximación a sus patrones de consumo, autopercepción y una caracterización de los sistemas productivos pecuarios de importancia en la alimentación y la economía familiar, se intenta ilustrar la importancia de la producción animal para el aporte de la seguridad alimentaría de las familias rurales del Sumapaz.

\section{PALABRAS CLAVE}

Seguridad alimentaría, producción pecuaria, campesinos, Sumapaz.

\section{FOOD SECURITY AND LIVESTOCK FARMING: THE CASE OF THE RURAL LOCALITY OF SUMAPAZ}

\begin{abstract}
The article discusses the issue of food security and livestock farming from the case study of the Sumapaz Locality, which belongs to the rural area of Bogota D.C. This territory is facing a serious food insecurity situation in accordance with information from previous studies. By approximating their consumption patterns, self-perception and a characterization of the importance of livestock production systems on food and household economy, this article attempts to illustrate the importance of animal production for food security of rural families of Sumapaz.
\end{abstract}

\section{KEY WORDS}

Food security, livestock production, farmers, Sumapaz. 


\section{INTRODUCCIÓN}

En la cumbre mundial sobre la alimentación de la Organización de las Naciones Unidas para la Agricultura y la Alimentación (FAO) que se celebró en 1996, se fijó la meta de reducir a la mitad el número de personas que padecen hambre para el año 2015 (FAO, 2006: 4). No obstante, y de acuerdo con la misma agencia, luego de doce años el panorama no sólo no ha mejorado, pues se mantienen la misma cantidad de personas hambrientas, sino que se le agregan nuevos elementos, como la actual crisis alimentaria, energética y económica que se configura en el entorno mundial.

Desde la óptica de los derechos humanos, el objetivo de esta lucha no sólo se puede centrar contra el hambre propiamente dicha, entendida como la sensación de desasosiego causada por la carencia de alimento, sino también contra la desnutrición y la malnutrición. En este sentido, es preciso anotar que en los países en vías de desarrollo, se tienen indignantes cifras de personas que no logran tener una alimentación adecuada. En particular, de acuerdo con las cifras disponibles, Colombia tenía un $13 \%$ de personas subnutridas para el año 1999 (DNP/PNUD, 2006: 37).

El país produce alimentos suficientes para suplir los requerimientos nutricionales de todas las personas. Sin embargo, la presencia de la desnutrición está explicada por el lado del acceso. Efectivamente, el debate de inseguridad alimentaria en Colombia, no puede concentrarse sobre la oferta, pues es una nación autosuficiente que cuenta con niveles crecientes de producción per cápita de alimentos. Por el contrario, la discusión debe darse en la demanda de alimentos, pues millones de personas no logran a acceder a niveles nutricionales adecuados para tener una vida sana (Machado, 2004: 34-40)

La pobreza es el determinante más importante de la subnutrición. De acuerdo con cifras del Departamento Nacional de Planeación en Colombia, a nivel nacional, la pobreza se sitúa en el $52,6 \%$, pero en las áreas rurales el $68 \%$ de la población vive en condiciones de pobreza y el $28 \%$ se encuentra en pobreza extrema. Los altos grados de pobreza en Colombia están estrechamente relacionados con los elevados niveles de desigualdad en la distribución de los ingresos, el cual es representado por el índice Gini $^{1}$, que para el país es del 0.56 , lo cual lo sitúa como el sexto país más desigual de América Latina (DNP/PNUD, 2006: 22-23).

Es paradójico que en las áreas rurales, donde precisamente se generan los alimentos, existan las peores condiciones de inseguridad alimentaria. Si bien las políticas públicas deberían estar destinadas a reducir los niveles de desigualdad y pobreza, como principal mecanismo para disminuir la inseguridad alimentaria, el drama humanitario que representa esta situación no puede esperar a que se alcancen estos objetivos. Por tal razón, la producción de alimentos de pancoger, representa el recurso más importante que tienen los campesinos, indígenas y afrodescendientes para satisfacer esta necesidad básica insatisfecha. 
La contribución de la producción pecuaria a la seguridad alimentaria de millones de pobladores rurales es muy importante, ya que los productos de origen animal son los alimentos que mayor proporción de proteína de alto valor biológico aportan a la nutrición humana, los cuales se pueden obtener parcialmente de sus propios animales domésticos.

Efectivamente, sólo para citar dos casos, se calcula que los campesinos colombianos producen el $40 \%$ de la leche que se consume en el país (Bejarano, 1998: 8; Forero, 2005: 15-16), y la producción campesina de huevos se estima en 3 millones de unidades diarias que abastecen el consumo de gran parte del área rural (Fenavi, 1998). De la misma manera, los excedentes de producción contribuyen de manera significativa a la economía de estas familias.

Para ejemplificar la situación de inseguridad alimentaria de los habitantes rurales, se escogió la Localidad del Sumapaz, en donde se realizó la investigación expuesta en el presente documento. En estudios realizados previamente por el Hospital Nazareth E.S.E Nivel I, entidad encargada de la salud de este territorio, para el año 2002 la desnutrición crónica ${ }^{2}$ alcanzaba el $47 \%$ y la aguda ${ }^{3}$ el $23 \%$. En niños menores de siete años la sumatoria de los porcentajes de desnutrición fue superior al $60 \%$, y en 105 infantes de 118 a los que se evaluó la albúmina sérica (proteína sanguínea medida para tamizar el consumo proteico), esta se encontraba por debajo del rango fisiológico normal (López y Casallas, 2004).

El presente documento tuvo como objetivo realizar una caracterización de los sistemas productivos pecuarios de la zona, su interrelación con la alimentación y con aspectos sociológicos, económicos y culturales del territorio, a fin de estimar el aporte de la producción pecuaria campesina a la seguridad alimentaria de las familias de la Localidad. Asimismo, se pretendió encontrar algunos elementos claves de intervención para programas de seguridad alimentaria en torno a los alimentos de origen animal.

\section{METODOLOGÍA}

La investigación fue hecha en la Localidad del Sumapaz, la cual hace parte del área rural de Bogotá D.C. Este territorio cuenta con 2.478 habitantes y se ubica dentro del páramo más grande del mundo. Cuenta con una extensión total de 78.096 hectáreas (Pérez, 2008: 63-66), la temperatura oscila entre los $4^{\circ} \mathrm{C}$ bajo cero hasta los $17^{\circ} \mathrm{C}$ y su altura está entre los 2.700 a los 4.560 m.s.n.m. (Dama, 2004).

El estudio se dio dentro del marco del proyecto de "Seguridad alimentaria para la familia rural del Sumapaz" del Hospital Nazareth E.S.E. Nivel I, entre el año 2005 y 2006. Para el logro de los objetivos propuestos, se aplicó una encuesta semiestructurada a 38 pobladores rurales que reportaron la tenencia de algún tipo de animal productivo, en una muestra por conglomerados para los tres corregimientos que tiene la localidad. La confiabilidad estadística del estudio fue del $90 \%$. El análisis de los indicadores se hizo mediante el programa estadístico SPSS. Asimismo, durante la ejecución del proyecto se tomaron datos etnográficos en notas de campo, para enriquecer la información cuantitativa. 


\section{RESULTADOS Y DISCUSIÓN}

\section{Características}

La modalidad de tenencia de la tierra está constituida en un $75.6 \%$ por propietarios (entre poseedores y propietarios con título), $18.9 \%$ aparceros y $5.4 \%$ arrendatarios. El $47.2 \%$ de los hogares encuestados manifestaron vivir hace más de quince años en las fincas. No obstante, es importante subrayar la migración rural-urbana que se da en la región por su asentamiento cercano a la ciudad de Bogotá.

El número de integrantes por familia es de 5.36 en promedio. Por su parte, el $43.2 \%$ de las fincas tienen más de 6 hectáreas (Gráfico 1) de las cuales el $69.4 \%$ está compuesto por un solo predio, y el $30.6 \%$ restante se encuentra conformado por dos y tres lotes no contiguos, normalmente en arriendo para pastoreo. El $66.06 \%$ del área está destinada a pastos, el $12.43 \%$ a cultivos agrícolas y el $17.56 \%$ está formado por bosques. De otro lado, el $79.7 \%$ de las familias cuentan con terrenos quebrados.

El $80 \%$ de los predios no sobrepasa las 20 hectáreas. El hecho de que la gran mayoría de los terrenos estén destinados a pastos para el ganado ha traído una gran deforestación, ya que los forrajes se establecen luego de la tala de bosques, sin consideración de la importancia ambiental que representa la riqueza hídrica del páramo del Sumapaz.

\section{Especies pecuarias y seguridad alimentaria}

La especie animal predominante en las familias de la Localidad es la bovina, con un $89.1 \%$ del total de las familias encuestadas, seguida por un $83.8 \%$ de aves, $45.9 \%$ de otras especies, $40.3 \%$ de porcinos y $35.1 \%$ de ovinos. (Gráfico 2 )

\section{Gráfico 1.}

ÁREA DE LA FINCA

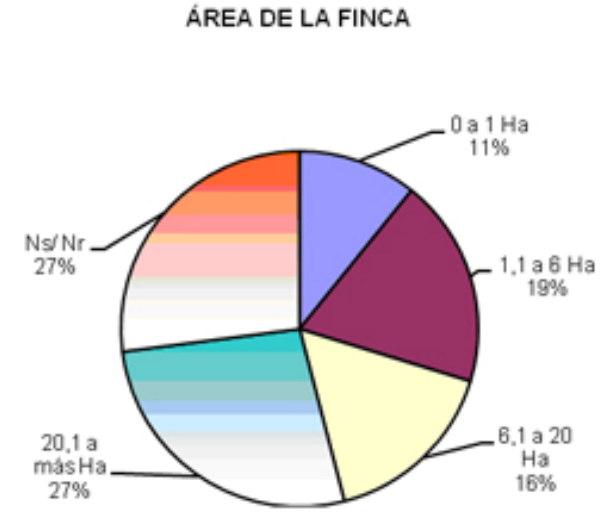

Gráfico 2.

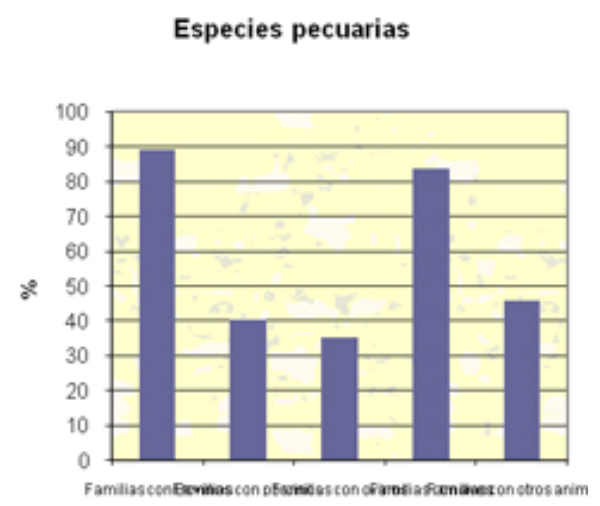

Fuente: Elaboración propia 
Según la opinión de los campesinos, la especie bovina es la más importante para los ingresos de su familia y la que consideran la más relevante para el autoconsumo del hogar, superando a especies menores que tradicionalmente se crían exclusivamente para el consumo interno. Las gallinas ocupan un puesto importante en la alimentación familiar, al proveer carne y huevos. Los encuestados ponen en un segundo orden de importancia a otras especies que eventualmente sacrifican para el consumo de carne (ovinos, pollos, etc.). En cuanto a ingresos, la segunda especie en importancia para las familias es la porcina, la cual es una fuente de ahorros supremamente importante, para gastos e inversiones que rutinariamente no se pueden hacer por falta de recursos.

Respecto a la relación del objeto de la producción animal en los hogares campesinos entrevistados, el $28.5 \%$ manifestó producir exclusivamente para el consumo y el $31.4 \%$ únicamente para el comercio. El $40 \%$ restante en una relación similar para consumo y para venta. Hay una correlación entre el tamaño de los predios y el destino de la producción. Entre más pequeña la unidad, es menor la cantidad de producto destinado para la venta, y entre más grande, los volúmenes para el comercio se hacen mayores.

La leche se consume diariamente en el $97 \%$ de los hogares que disponen de animales, en un promedio de 0.64 litros por persona/día. Las recomendaciones per cápita de la OMS son mínimo de $0.4 \mathrm{~L}$ /día. El $46 \%$ de los encuestados dejan un excedente del queso para su consumo: en promedio 7.6 libras por semana. Entretanto, los huevos se consumen en un promedio de 4.7 unidades a la semana por persona, de los cuales el $87 \%$ los proporcionan los animales de la granja.

La carne tan sólo manifiestan consumirla el $16.2 \%$ de las familias y el $62 \%$ no consume alimentos diferentes de origen animal el fin de semana, lo cual revela una dieta muy rutinaria.

En las celebraciones especiales, el $54 \%$ sacrifica gallinas o pollos. El $40 \%$ consume carne de res. Para muchas familias estas son las únicas ocasiones en las que se alimentan con algún tipo de carne. Animales como cerdo, cordero o chivo se consumen respectivamente en el $21 \%, 19 \%$ y $5.5 \%$ de las familias. El $13.5 \%$ de las familias pesca trucha para fechas muy especiales y el $2.7 \%$ de las familias celebran fechas especiales con huevos. Por otro lado, tan sólo el $14.2 \%$ intercambia productos de origen animal con sus vecinos. La caza la realiza el $14.2 \%$ de los consultados 4 veces en promedio al año, principalmente animales como conejo, borugo y cuy.

\section{Autopercepción}

Se procuró evaluar en las familias la percepción de su entorno y de su seguridad alimentaria, como pilar indispensable a partir del cual se debe dar inicio al trabajar con una comunidad en proyectos que impulsen el mejoramiento de su nutrición.

El $54 \%$ de las personas consideraron no poder mejorar su condición de vida con los recursos disponibles. Son, en efecto, campesinos pobres, que viven del trabajo a 
jornal y con poca tierra. El $45 \%$ restante, manifestó que tiene los recursos suficientes para mejorar su alimentación y calidad de vida, conformando el grupo que realmente cuenta con los mayores medios. El $56.7 \%$ de las personas piensa que su situación actual es mejor que la de muchas personas.

Ante la pregunta de cuál era el mayor limitante para el desarrollo de la producción pecuaria de su finca, el mayor porcentaje $(46 \%)$ se inclinó por la falta de tierra suficiente. Esto es obvio cuando las personas piensan en ganado. Sin embargo, si consideramos que el $43 \%$ de las personas tienen más de 6 hectáreas, puede pensarse en el desarrollo pecuario con producciones intensivas de otras especies menores como las gallinas. La falta de dinero compartió el primer lugar. Muchas personas, aunque tienen acceso a la tierra y disponibilidad de mano de obra, no poseen capital, haciéndose necesario brindar créditos blandos o subsidios para la producción de sus terrenos junto a una juiciosa asistencia técnica. Esta última opción fue considerada por el $29.7 \%$ de las personas. (Gráfico 3).

\section{Gráfico 3}

\section{Cuales son los mayores limitantes para el desarrollo pecuario de la finca}

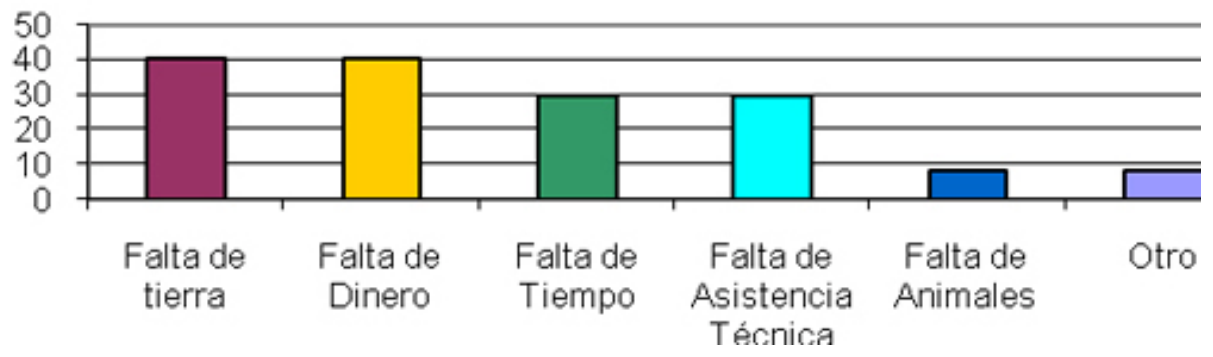

Fuente: Elaboración propia

Cuando se preguntó de quién deberían partir las soluciones para el desarrollo pecuario de su propia finca, el $46 \%$ respondió que de ellos mismos. Al Estado se la adjudicó el $40.5 \%$ de las familias. Por su parte, ante la pregunta de cómo debería ayudar el Estado, el $60 \%$ de la población escogió la opción del suministro de semillas, insumos y animales. El $40 \%$ de los encuestados escogió la opción de prestación de capacitación técnica. Muchos entrevistados eligieron esta última alternativa, junto al suministro de insumos.

El 21.6\% dijo que le gustaría que el Estado apoyara procesos de autogestión de la comunidad. Este sería el punto decisivo de los programas de seguridad alimentaria, cuando las comunidades se empoderen y articulen la producción, la cohesión social, el intercambio de productos y conocimientos, de tal forma que se entrelace el tejido humano para que las redes de solidaridad comunitarias gestionen su propio desarrollo. 
Analizando los cambios que ha tenido su alimentación en los productos de origen animal con respecto a su infancia o la de padres y abuelos que vivían en la región, el $54 \%$ dijo que ahora se come mucho mejor que antes, debido a la parcelación que fue posible luego de los conflictos agrarios que se dieron a comienzos del siglo XX. En especial, en lo relacionado con sus hábitos alimentarios, una persona octogenaria, de la época de la gran hacienda, manifestó que de niño comía las socas de cosecha de papa y diferentes tipos de desperdicios agrícolas; otras con tristeza, dijeron que su infancia había sido supremamente pobre y que ahora su alimentación es notablemente mejor. El 19\% expresó que antes se cazaba mucho más. De hecho, muchos animales silvestres que ahora no se ven, como la danta y el oso de anteojos. Por su parte el $8.1 \%$ explicó que antes comía mejor, probablemente su situación económica haya empeorado. El 10.8\% manifestó consumir en el pasado más carne y el $2.7 \%$ pescar más que ahora.

\section{Sistemas productivos pecuarios}

Son tres los factores determinantes para lograr la seguridad alimentaria: la disponibilidad de los alimentos, el consumo y la utilización biológica de los mismos. En esta sección se hace una caracterización de los sistemas pecuarios de la región, con el fin de realizar un diagnóstico productivo y su relación con el consumo.

Una tecnología muy importante para el desarrollo del sistema productivo bovino ha sido la incorporación de la cerca eléctrica. En la zona, tan sólo el $13 \%$ de los encuestados carece de este tipo de cerca en sus potreros. Sin embargo, por la falta de capacitación técnica, existe un sobrepastoreo excesivo, llegando al extremo de pastoreos continuos de dos meses, lo cual obliga a posteriores recuperaciones de cuatro a seis meses.

El sistema productivo de la región es el doble propósito. Solamente en el $30 \%$ de las fincas se suplementa y en forma bastante esporádica; la sal mineralizada se suministra únicamente en el $9 \%$ de las fincas. Esto conlleva a problemas reproductivos y a enfermedades como retención placentaria. La producción promedio de leche estimada por los entrevistados es de 5.5 litros promedio vaca/día, lo cual es un parámetro aceptable, comparada con otras regiones del país, teniendo en cuenta, además, la ausencia de suplementación diaria a los animales

La edad vs peso al momento del sacrificio para carne, es supremamente deficiente, debido al pésimo manejo de novillos, las malas praderas del páramo y la altísima incidencia de enfermedades parasitarias, en especial la Fasciola Hepática que se perpetúa por las deficientes prácticas sanitarias profilácticas de la región. En el $97 \%$ de los predios no se realiza curación de ombligos de terneros, lo que lleva a enfermedades neonatales que los campesinos reportan como comunes.

Por otro lado, el $76 \%$ de familias compran lechones criollos para engorde, los cuales son alimentados con subproductos como hollejo de papa, desechos de cocina y suero de leche. Es el sistema de producción más rentable de la región ya que no le representan egresos a los hogares. Transferencias de tecnologías tan simples como 
la construcción de cocheras artesanales para resguardarlos del frío, mejores planes de vacunación y desparasitación, y prácticas tan sencillas como cocinar algunos subproductos, redundarían en mayor productividad. Por su parte, el $100 \%$ de las familias alimentan las gallinas con maíz amarillo, y el $48 \%$ tiene a los animales libres, en edades mezcladas y ambos sexos, sin ninguna instalación.

Respecto a la inocuidad alimentaria, el uso indiscriminado de medicamentos, en especial antibióticos como la oxitetraciclina, o purgantes como el albendazol, sugiere una residualidad en alimentos como la leche y los huevos, con riesgos de resistencia de estos productos, tanto en la misma población como en las zonas urbanas en donde se mercadea el producto. La falta de asistencia técnica en estas economías ha conllevado a problemas importantes en salud pública que sobrepasan las barreras geográficas de las zonas campesinas.

\section{Perspectiva de género}

Los hombres hacen solos las actividades propias del manejo del ganado en el $31 \%$ de los casos, acompañados de sus hijos, principalmente varones, en el $12,5 \%$ de las familias. Sin embargo, estas labores se hacen en conjunto con sus esposas en el $22 \%$ de los ocasiones. Las tareas que demandan las lecherías, entre otras, son apartar el ganado, rotar praderas, ordeñar y prestar asistencia sanitaria.

En este apartado se puede concluir que las mujeres participan en una menor proporción en el manejo directo del ganado. No obstante, realizan estas actividades cuando no hacen parte de la fuente de ingresos principal de las familias en el 9,4\% de los hogares encuestados. Con respecto a la transformación de la leche en quesos y cuajadas para consumo de la familia y la comercialización, las mujeres efectúan esta actividad en el $87 \%$ de los casos, y son acompañadas por sus maridos únicamente en el $8,3 \%$ de las ocasiones. La anterior es una actividad productiva, pero que es más reconocida como una labor reproductiva en el área rural.

Si se analiza el ciclo productivo de los derivados lácteos que, según en el mismo estudio son considerados por los habitantes en el $44 \%$ de los casos como la principal fuente de ingresos en conjunto con la venta de carne, el trabajo de las mujeres es más integral que el de los varones de la región. Las mujeres combinan las actividades de cuidado de los animales y ordeño solas o en compañía de sus maridos e hijos, pero trabajan sin su ayuda en la transformación del producto.

Con respecto al manejo de especies menores, como cerdos, que son fuente de ahorro para las familias para fin de año, las mujeres aportan su trabajo alimentándolos, criando y prestando cuidado general en el $50 \%$ de los casos, frente al $6,7 \%$ de los hombres. Si se analiza el cuidado de aves como pollos y gallinas para la venta y consumo al interior de los hogares, las mujeres se dedican solas en el $57,1 \%$ de los casos, mientras que los varones solos realizan esta actividad en el $4,8 \%$ de las ocasiones. 
Las anteriores son evidencias que reflejan la integración cada vez más creciente de las mujeres a las actividades productivas de los hogares rurales y a la seguridad alimentaria de sus propias familias. La nueva ruralidad trae transformaciones que muestran un rompimiento en la distribución del trabajo productivo en las familias, pero que aún no logra cambios de fondo en el rol de los hombres en las actividades reproductivas de las áreas rurales colombianas.

\section{Ingresos}

El $71.4 \%$ considera insuficiente sus ingresos económicos. La mayor fuente de estos ingresos lo constituye el trabajo por fuera de su unidad productiva, bien sea por jornal, en especial en labores de agricultura, en cultivos de papa o en trabajos no agropecuarios, como evidencia de una nueva ruralidad. El segundo renglón en importancia lo ocupa la producción pecuaria. Los ingresos pecuarios representan en el $62 \%$ de los casos menos de la cuarta parte del total de ingresos. El $20.6 \%$ vive casi exclusivamente de este renglón productivo. El resto combina actividades que reflejan la pluriactividad de las economías campesinas.

\section{RECOMENDACIONES}

A partir de los datos encontrados se pudo concluir el enorme aporte que tiene la producción animal para la seguridad alimentaria de los habitantes del área de estudio. La leche y derivados lácteos, así como los huevos, son los alimentos más importantes para el autoconsumo de los habitantes del Sumapaz. La venta de quesos y cría de cerdos son actividades que generan ingresos económicos importantes a las familias para adquirir el resto de la canasta básica de alimentos. Se evidenció, además, el gran aporte de las mujeres en la producción pecuaria y la seguridad alimentaria de las familias.

Se sugiere realizar sensibilizaciones del problema nutricional y actividades comunitarias que conlleven a la articulación del tejido humano, para incentivar el trueque, y el intercambio de técnicas productivas, sanitarias y culinarias. Asimismo, aprovechar al máximo el acceso a la tierra, recurso que se debe optimizar ojalá en explotaciones intensivas con especies menores para la alimentación de las familias. Se sugiere dar inicio a un proyecto de producción de gallinas en semipastoreo, con leguminosas y cultivo de lombrices como fuente de proteína. En efecto, el huevo es la proteína más accesible y barata como sustituto de la carne. El acceso se debe complementar con programas de culinaria, para complementar la alimentación de las familias.

Se recomienda estimular el consumo de leche, con talleres de elaboración de productos lácteos, en especial yogures con frutas silvestres como la mora o el mortiño. Por otro lado, se sugiere dar un mayor énfasis al trabajo pecuario de seguridad alimentaria con las mujeres rurales. La realización de talleres culinarios con base en recetas balanceadas, con productos que se produzcan en la huerta casera y en toda la finca, sería una gran alternativa para recobrar el placer de comer y atacar la rutina de la dieta. 
Hacer capacitaciones sobre manejo de praderas, prácticas para la estimular la alimentación del ganado con ensilaje de papa y suplementación con sal mineralizada. Entrenamientos sobre higiene del parto, curación de ombligos, planes preventivos de enfermedad y mejoramiento de la rutina de ordeño, con el fin de mejorar la calidad higiénica de la leche, son algunas de las transferencias de tecnologías importantes que necesita la región

A los entes gubernamentales se recomienda patrocinar programas de seguridad alimentaria que incluyan capacitación técnica junto al suministro de insumos, especialmente para familias muy pobres, con un gran contenido de acción sociológica y vigilancia nutricional. Así como ofrecer facilidades en programas de subsidios y créditos blandos $\mathrm{y}$, finalmente, reconocer la gravedad del problema nutricional en todo el país, dándole el puesto de importancia que debe ocupar en las políticas públicas, con la asignación de partidas económicas que merece el drama nutricional en regiones como la expuesta y algunas alternativas sugeridas en el presente documento.

\section{AGRADECIMIENTOS}

El autor expresa sus agradecimientos al Hospital Nazareth E.S.E. Nivel I, al profesor Alonso Correa de la Universidad Nacional de Colombia, a los ejecutores del proyecto de seguridad alimentaria y a toda la comunidad sumapaceña que prestó su tiempo y experiencia para hacer posible este trabajo.

\section{BIBLIOGRAFÍA}

- Bejarano, Jesús. 1998. Economía de la agricultura. Universidad Nacional de Colombia. Tercer mundo. Bogotá. D.C, Colombia.

- Dama. 2004. Diagnóstico de la situación actual y condiciones de los sistemas de comercialización de los principales productos agropecuarios del área rural de Bogotá D.C. Bogotá. D.C, Colombia.

- FAO. 2006. El estado de la inseguridad alimentaria en el mundo. Roma. Italia.

- Fenavi. 1998. El mercadeo del huevo en Colombia. Cuadernos avícolas. Universidad Jorge Tadeo Lozano. Bogotá. D.C, Colombia.

- Forero, Jaime. 2002. Economía campesina y sistema alimentario en Colombia. Aportes para la discusión sobre seguridad alimentaria. Pontificia Universidad Javeriana. Bogotá D.C. Colombia.

- López, Mónica; Casallas, Maricela. 2004. Seguridad alimentaria de la familia rural del Sumapaz. Tesis. Universidad Nacional de Colombia. Bogotá. D.C.

- Machado, Absalón. 2004. Seguridad alimentaria y sistema agroalimentario. En: Territorios y sistemas agroalimentarios localizados. Universidad Nacional de Colombia. Bogotá D.C.

- Pérez, Manuel. 2008. La adaptabilidad de pobladores y asentamientos rurales en áreas de conurbación: El caso de la ciudad de Bogotá (Colombia). En: Cuadernos de desarrollo rural (59): 11-3. Bogotá D.C. Colombia. 


\section{NOTAS}

1. El índice Gini es una escala de 0 a 1 , en la cual 0 significa que los ingresos se distribuyen de manera totalmente igualitaria y 1 que todos los ingresos se concentran en los más ricos.

2. Desnutrición crónica: Talla Vs. Edad

3. Desnutrición aguda: Talla Vs. Peso 